\title{
Microstructured porous material models for damage induced by fluids in compressed rocks
}

\author{
Anna Pandolfi \\ Civil and environmentalengineringDepartmente \\ Politecnico di Milano \\ Piazza Leonardo da vinci 32, 20133 Milano Italy \\ e-mail:anna.pandolfi@polimi.it
}

\begin{abstract}
A multiscale brittle damage model is used to simulate the behavior of confined rock materials in laboratory tests. The material model is characterized by microstructures in the form of recursive equidistant parallel cohesive/frictional faults, and it is defined by a reduced number (seven) of material constants. Simulations show that the model is able to capture several important features observed in rocks, in particular the reduction of the overall stiffness for increasing deterioration of the material, fragile to ductile transition, strain localization, shear band formation, and more general size-effect. The model, extended to coupled hydromechanics, has been applied to the simulation of the experimental tests of rocks at the material point scale, at the laboratory specimen scale, and at the full field scale. The model shows good predictive properties for a large number of geo-materials, in particular it is able to establish a correspondence between the actual microstructure and the macroscopic geometry of shear bands.
\end{abstract}

